\title{
Co-evolution of Diplomacy after the Corona Crisis: An Agenda for Practitioners
}

\author{
Dalya Salinas Pérez, ${ }^{a}$ Ulises Canchola Gutiérrez ${ }^{b}$ and José-Juan \\ López-Portillo ${ }^{a}$ \\ aEmbassy of Mexico to the Kingdom of Norway, Oslo, Norway \\ bAmbassador of the United Mexican States to the Kingdom of Norway, Oslo, \\ Norway \\ dsalinas@sre.gob.mx; ucanchola@sre.gob.mx;jjlopezp@sre.gob.mx
}

Received: 18 June 2020; revised: 23 September 2020; accepted: 29 September 2020

\section{Summary}

The 'corona crisis' has transformed a generalized and long-standing concern for foreign service reform into an urgent necessity. The reform of the Mexican Foreign Service Law in 2018 offers a valuable example of a recent, comprehensive attempt to prepare a diplomatic apparatus for a context of accelerated change and uncertainty. The authors of this essay, who directly participated in this reform, explain how some of its main features provided them with useful tools to respond effectively to the COVID-19 pandemic from their new posting in Norway. After discussing the lessons from their experiences, they propose an agenda for discussion among practitioners and academics on subjects that must be addressed if diplomacy is to fulfil its urgent role in the construction of a new, post-pandemic world order.

\section{Keywords}

diplomacy - professionalization - reform - evolution - ecosystem - diplomatic innovation 
In 2018, two of us were directly involved in drafting a proposal for what has become the most comprehensive reform of Mexico's Foreign Service Law (MFSL) in more than twenty years. Our endeavour was precipitated by a simple, if long-standing, observation: diplomatic practices have remained anchored in an inertia that has made them resistant to evolving with the times. An unprecedented degree of interconnection driven by exponential technological innovation was engendering a disruptive Fourth Industrial Revolution. Despite our interconnectedness, however, uncertainty predominated and there was no consensus on how to address global challenges. Zygmunt Bauman's essay Liquid Modernity seemed to capture the complexity of the international context accurately: traditional power structures were everywhere contested, any consensus appeared short-lived, social solidarity and collaboration were being degraded, knowledge and long-term thinking were collapsing. ${ }^{1}$ In a context of radical uncertainty, we could not presume to forecast how diplomacy should look in twenty years. Instead, the underlying challenge was to reform the law in such a way that it would allow diplomatic practice to update itself to face unforeseeable challenges.

A symptom of the erosion of traditional power structures was that diplomats appeared as increasingly irrelevant and out of touch with new realities. Colleagues from at least five countries shared their experiences in trying to strengthen their foreign services following a crisis in which their diplomatic response had proved ineffective. However, the emergence of a 'ubiquitous diplomacy', in which non-state actors contested the state's traditional initiative in defining global governance outcomes, was a sign of diplomacy's pertinence. The diplomatic profession had to evolve and adapt to 'liquid times' if 'the bringing of power and politics together again'2 was to be successful.

The corona crisis has evinced most elements of our prognosis, providing an opportunity to test our hypothesis and extrapolate valuable lessons for professional diplomacies around the world.

In 2018, we seized on a rare political juncture. The Ministry of Foreign Affairs (MFA) and all political parties in the Mexican Congress reached a political

1 Bauman 2018.

2 Bauman 2018, 25. 
consensus in the final months before the end of the legislative period. They agreed on the importance of strengthening the Mexican Foreign Service to better equip our country to navigate uncertain times. Making the most of the political will one could count on to promote a reform of the MFSL in a compressed time frame, we focussed on identifying mechanisms that would allow Mexican diplomacy to adapt and evolve with a rapidly changing global context.

Despite the time constraints, we envisaged the process of reform as an exercise in transparency. All members of our Foreign Service were invited to make comments and suggestions or raise questions regarding different aspects of the law. Their accumulated experience benefitted the reform enormously. We also derived invaluable expertise from other Mexican departments of state and autonomous organisations such as the Central Bank. We complemented these national perspectives with insights gleaned from other foreign services, international experts in diplomatic studies and international consultants.

\subsection{Systemic Diplomacy: Open a Closed Club}

Aware of the inward-looking tendencies of any bureaucracy, the reform of the MFSL expanded three pre-existing mechanisms to expose our diplomats to influences beyond our Foreign Service. MFA authorities were granted the faculty to commission any of its members (of either branch - diplomatic or administrative - regardless of rank) for up to three years to an external organisation within the public sector, academia, international or civil society. We hoped thereby to cross-pollinate knowledge and expertise between professional diplomats and other sectors of government and society in general.

Second, under Article 7 of the MFSL, the reform safeguards, but standardises and regulates, temporary appointments of eligible external experts to participate in the Foreign Service for up to six years. It is worth emphasising the relevance of Article 7. Within its scope, specific profiles were developed to recruit experts on various topics. A new approach was taken on science and technology. The new profile would allow an external recruit to serve as a 'pathfinder' in epistemic communities, a role we deemed as essential for diplomacy to engage with relevant actors in emerging agendas related to the knowledge economy; namely, technology and innovation.

Finally, the reform enables the Foreign Service Chairmanship to assess the profiles of personnel from other ministries that will be deployed as attachés in embassies and consulates abroad. This measure aims to provide technical experts with basic diplomatic knowledge, as well as to facilitate their understanding and harmonious integration into the dynamic of a foreign mission. This also provides for better co-ordination among ministries through improved 
communication aimed at fostering mutual understanding and, most importantly, cohesiveness of professional diplomacy.

\subsection{Diplomatic Professionalisation in a Context of Uncertainty}

Our MFA's authorities considered that Mexico needed a diplomatic apparatus that corresponded to its expanding geopolitical role. Professionalisation is essential in this regard, because it sets standards and thereby establishes a framework through which to evaluate performance that, in turn, allows improvements designed to increase effectiveness.

In contexts characterised by rapid change and uncertainty, the solution is not to deprofessionalise nor is it to lurch towards the opposite extreme of insisting on rigid controls. A hybridised image that allows our professionals 'to focus on new connections between work, organisational action, and outside worlds, as well as on how these connections are made meaningful' 3 encapsulated what the reform sought by design. Three elements would constitute our new, hybridised, professional diplomacy: a career plan with a reasonably short shelf life, a new methodology for evaluating performance and a subcommittee within the Foreign Service Commission dedicated to the continuous assessment and adjustment of the aforementioned mechanisms, as well as the generation of prospective studies on the matter.

A common thread running through our proposal was the need to reinvigorate the diplomatic academy, Instituto Matías Romero (IM R). This involved increasing its decision-making role in the Foreign Service Commission, as well as enabling it to design courses aimed at audiences other than the MFA. We intended to improve diplomatic performance but also to elevate the standards and quality of training programs based on multidisciplinary convergence in the classroom. In that sense, the IM R could serve as a window through which outsiders could gain an insight into the unknown or misunderstood world of diplomacy, while diplomats could glean insights from external experts. By transforming the source of our professionalisation into a multidisciplinary space for thinking about our career as a process of dynamic evolution, we hoped to promote a mentality among professional diplomats that embraces accelerated change and adaptation.

\subsection{Inclusiveness and Diplomacy: Empowering Our Capacities}

A forward-looking reform to the MFSL could not neglect the urgent need to embrace gender parity, inclusion and diversity. We realised that a diplomatic

3 Noordegraaf 2007, 776. 
system integrated by a more diverse group of professionals would be better prepared and more resilient than one which promotes only a homogenous perspective. Diversity and the recognition of the importance of non-traditional or 'soft skills' constitute key strengths in conducting diplomacy. The MFA's responsibility is to attract this type of talent and nurture it to retain it for decades. Diversity strengthens diplomatic capacities and allows us to represent the interests of the societies we serve more completely. This is also part of a necessary evolution of our profession.

We could not change unconscious biases or entrenched attitudes by law but we could use its provisions to nudge behaviour towards a more welcoming organisational culture. For instance, the reform granted Heads of Mission the faculty to implement flexi-work arrangements to help colleagues under their care to better reconcile the demands of professional and domestic work and it allocated new financial resources towards providing subsidies for day-care services and longer paternity leave. To embed these culture changes, we included the requirement that any governing body and decision-making process had to reflect gender parity. Furthermore, the Gender Equality Unit of the Ministry of Foreign Affairs was added as a member of some of the subcommittees of the Foreign Service Commission.

\section{The Reformed Diplomacy in Light of the COVID-19 Pandemic}

As the MFSL begins its process of implementation in a context of crisis provoked by the COVID-19 pandemic, our underlying prognosis seems increasingly justified: diplomacy should focus on adapting to uncertainty. Our experiences during the pandemic suggest that the best way to achieve this is through increasing the range of our interactions and allowing inputs from diverse experiences to update our practices.

Our posting to Norway in mid-2019, allowed us to use the new regulations governing Article 7 to recruit an external expert that would be in charge of engaging with Norwegian epistemic communities and a variety of stakeholders in its innovation ecosystems. By launching this unexplored agenda, our team had to adopt a more agile work culture and became accustomed to processing large amounts of highly technical information, which we could then transmit as meaningful reports to our capital.

As a result, by the time the WHO declared that we were facing a pandemic, our team could react with relative speed. Through the unconventional networks we had begun to create, we quickly became familiar with the Coalition 
for Epidemic Preparedness Innovations (CEPI) and were able to anticipate the centrality of its role in international efforts to overcome the pandemic. We conveyed the necessary information to our capital and advocated successfully for Mexico to join CEPI. Thereafter, we had to react quickly to the exigencies of membership in CEPI - an innovative multi-actor coalition - as well as the broader agendas of the wHо's Аст-Accelerator, of which it forms part. Some of our experiments with working transversally and using new digital platforms made it relatively easy to transition to work away from the office during periods of lockdown or quarantine, while simultaneously continuing to pursue our pre-CEPI activities. Nevertheless, we acknowledge that we are still far from establishing common norms of digital interaction that will allow us to replicate physical meetings: not only do the technical capabilities of different interlocutors vary but we have not found a way to replicate valuable forms of communication that create confidence between interlocutors such as informal conversations after a meeting, sharing refreshments or exploiting chance encounters.

As we adapted to our new role as members of CEPI, we realised that we were mimicking techniques traditionally associated with three professions: first, like investigative journalists, we had to gain access to better than average information, by which we mean it had to be more accurate and up to date than that which is commonly available online or through other public sources. Second, like strategy consultants, we were analysing the information we gathered with the objective of making proposals for concrete courses of action to our capital. Finally, we began to mimic brokers and other intermediaries who create relations of confidence with their clients, match their interests to those of other actors and thereby limit their risk to lower transaction costs that can lead to better-than-average results.

Our tactics evolved to respond to a substantive need: the race to develop and manufacture vaccines was not and could not be treated as a unidimensional problem based on purely technical considerations or market forces. Our analysis had to include a range of factors such as political considerations related to promoting an agenda of global co-operation and equitable access, or assessing the value of vaccines over time when viable alternatives of uncertain feasibility (herd immunity, therapeutics, face masks, etc.) could emerge. We had to evaluate which vaccine candidate might be the most useful, given Mexico's demographics, as well as analyse the strength of our health system in relation to a multiplicity of social and economic factors involving issues as diverse as comorbidities, mental health and cultural norms associated with gender and age for care at home. The variables involved justified our insistence in the reform 
that an effective diplomatic strategy should be able to identify and include advice from a range of external interlocutors - in this case, those specialised in immunology, statistics, mathematical modelling and social sciences - to that of professional diplomats capable of translating hard evidence into recommendations or tactics for negotiation. Our experiences also underscored the importance of including a range of perspectives that were more representative of our home societies — including gender and socio-economic ones — in the formulation of our recommendations.

Provisions encoded in the reform could allow the Mexican Foreign Service to adapt to these new exigencies. For instance, lessons from our Embassy and other missions' experiences can now be used by the Analysis and Prospective Sub-committee to improve training programs and the Foreign Service career plans. Diplomats can be commissioned to key organisations, building bridges that favour information flows and mutual support, even with the private sector. Administrative innovations in favour of inclusion and diversity, such as day-care subsidies and the faculty to implement flexi-work arrangements, provide professionals with better conditions to be competitive vis-à-vis other countries or stakeholders.

Our experiences during the pandemic have illustrated some of the potential avenues that Mexican diplomacy could pursue thanks to the reformed MFSL. We are aware, however, that the letter of the law serves only as a framework or a first step. To realise the reform's potential, we will need to implement its provisions with the spirit that animated its composition. A new type of diplomat is needed: one who can connect and operate with increased agency and initiative in a more diverse, uncertain and risky environment. At the same time, MFAs must also provide diplomats with the confidence to offer feedback and engage in advocacy, as well as self-assurance for their attempts to advance novel agendas, leadership styles and tactics.

The global scope of the CoviD-19 crisis has demonstrated, in stark terms, the essential value of diplomacy in shaping a more resilient and prosperous world. The profound disruptions caused by this crisis constitute an opportunity to identify where diplomacy must evolve to fulfil its role with renewed effectiveness. In the case of the Mexican Foreign Service, we were fortunate that contingent political alignments gave us the opportunity to formulate an early diagnosis of the volatility inherent in a highly interconnected world and to 
devise a response in the reform of the MFSL. By its very nature, reform is an ongoing process. The current crisis makes reflecting on diplomacy on a more global scale an urgent matter.

In the reform, it is proposed that diplomacy should aim to become a more inclusive, knowledge-based network of professionals and institutions. It makes sense, therefore, to work towards a global agenda for practitioners, supported by academic research, that profits from this crisis by promoting a better understanding of diplomacy as a profession, as well as its role and public worth in the pursuit of global solutions.

A preliminary reading of the diplomatic response to the crisis makes it essential to discuss the following agenda:

- The future of our profession relies on its capacity to generate public value under conditions of uncertainty, high risk and rapid change. To that end, our traditional institutions and social structures cannot endure as our main points of reference when thinking about how to adapt. Instead, we should be oriented by our networks. That is how we would deal with the 'collapse of long-term thinking' referred to by Bauman. ${ }^{4}$

- Professional diplomacy should open itself to external influences. If we equate diplomatic professionalisation with an ability to coevolve with other actors in a multi-stakeholder ecosystem, diplomacy can incorporate new skills, develop more agile practices and replicate knowledge. Moreover, diplomats can use this learning to enhance the level of understanding within their professional sphere and bring it to their interactions with other relevant actors. As a result, diplomacy can be more influential, effective and cohesive.

- An agenda that promotes diversity, inclusion and a gender perspective into MFAs should be an end in itself. In a context of radical uncertainty, such an agenda becomes indispensable. It is only through diversifying perspectives that MFAs can prepare themselves to face a greater range of variables with greater sophistication and to address their complexity with the greatest probability of success. Furthermore, the ethos of inclusion implies an openness to adaptability as it induces individuals to change their minds and accept or tolerate diversity. It also promotes a principle of solidarity between sectors of society that can otherwise interact only through asymmetrical power relations that have come to define our 'liquid modernity'. By incorporating this ethos into MFAs, we prepare our diplomats to think in terms that transcend mere power and competition, privileging solidarity, dialogue and care instead, which are the values that offer the best hope of overcoming global challenges.

4 Bauman 2018, 3. 
- The covid-19 crisis has accelerated the rate at which diplomats adopt digital tools. However, this has diminished diplomatic capacities for two main reasons: first, existing digital platforms are unable to replace the scope of communication provided by personal encounters. Second, diplomats are becoming increasingly dependent on digital platforms developed for commercial interests. To fulfil its mission, diplomacy must embrace a strategic approach to engaging with existing technologies and their developers. By this, we do not mean practicing 'digital diplomacy' as it is commonly understood. It is not a matter of communicating with audiences through social media platforms. It is about acknowledging that such 'public diplomacy' never takes place in a neutral sphere. Instead, commercial digital platforms aim to maximise the profits of corporations through algorithms that determine the transmission of messages to a purposefully fractured public sphere. A strategic approach should involve strengthening diplomatic comprehension of technology, not just technological products. In doing so, it is co-creating both digital public goods better suited to the agendas and societies that diplomats serve, as well as adequate tools for diplomatic work. More broadly, to regain the initiative in the technological sphere, diplomats need to be aware of the general trends of the exponential technological change we are witnessing. This will give them a better chance to respond to its disruptions and to provide strategies to redirect them towards generating public value.

Part of our co-evolution with technological change will necessarily depend on understanding the needs and objectives of diplomatic practice and how technology can and cannot help to fulfil them. Diplomacy is too important to rely on off-the-shelf technological solutions.

Underlying our proposals is the need for a better understanding of the nature of diplomacy and what makes it such a powerful tool, particularly in the current context of global change of epoch. As we reflect on this problem, we should not shy away from developing new protocols and practices that are better suited to our times of uncertainty and change.

\section{Bibliography}

Bauman, Zygmunt. Liquid Modernity (Cambridge: Polity Press, 2018).

Noordegraaf, Mirko. 'From "Pure" to "Hybrid" Professionalism: Present-Day Professionalism in Ambiguous Public Domains'. Administration and Society 39 (6) (2007), $761-785$. 


\section{Other Sources}

Constantinou, Costas M., Noé Cornago and Fiona McConnell. 'Transprofessional Diplomacy'. Diplomacy and Foreign Policy (1) (4) (2016), 1-66. DoI 10.1163/ 24056006-12340005.

Fletcher, Tom. The Naked Diplomat: Understanding Power and Politics in the Digital Age (London: William Collins, 2017).

Foa, R.S., A. Klassen, M. Slade, A. Rand, and R. Collins. 'The Global Satisfaction with Democracy Report 2020' (Cambridge: Bennett Institute for Public Policy, University of Cambridge, 2020). https:/www.bennettinstitute.cam.ac.uk/media/uploads/ files/DemocracyReport2o2o_nYqqWio.pdf.

Hanson, Jonathan K. and Rachel Sigman. 'Leviathan's Latent Dimensions: Measuring State Capacity for Comparative Political Research'. APSA (2019). https:/qog.pol .gu.se/digitalAssets/1733/1733772_hansonsigman_lld_201906o5.pdf.

Mazzucato, Mariana. The Entrepreneurial State: Debunking Public vs. Private Sector Myths (London: Anthem Press, 2013).

Moore, Mark. Creating Public Value: Strategic Management in Government (Cambridge: Harvard University Press, 1995).

Murray, Stuart, Paul Sharp, Geoffrey Wiseman, David Criekemans and Jan Melissen. 'The Present and Future of Diplomacy and Diplomatic Studies'. International Studies Review 13 (4) (2011), 709-728.

Qin, Yaqing. 'Diplomacy as Relational Practice'. The Hague Journal of Diplomacy 15 (1-2) (2020), 165-173. DOI 10.1163/1871191X-15101092.

Ricciuti, Roberto, Antonio Savoia, and Kunal Sen. 'What Determines Administrative Capacity in Developing Countries?' International Tax and Public Finance 26 (2019), 972-998.

Sharp, Paul. 'For Diplomacy: Representation and the Study of International Relations'. International Studies Review 1 (1) (1999), 33-57.

Sharp, Paul. Diplomacy in the 21st Century: A Brief Introduction (London: Routledge, 2019).

\section{Dalya Salinas Pérez}

has been a member of the Mexican Foreign Service since 2006. She has served in the General Consulate of Mexico in Nogales, Arizona (2006-2008), in the Embassy of Mexico to the Kingdom of the Netherlands (2011-2015), and is currently Head of the Consular Section at the Embassy of Mexico to the Kingdom of Norway (since August 2019). She was appointed as an advisor to the Foreign Minister's Office (2009-2011; July 2015-March 2016), Director for Political Affairs and UN General Assembly in the General Directorate for the United 
Nations System (March 2016-October 2017) and Deputy Director General at the Chairmanship of the Personnel Commission of the Mexican Foreign Service (October 2017-July 2019). She graduated with honours from the Bachelor of Arts Program in International Relations from IтAM (Mexico City, 2006), and holds a master's degree in international communication management from The Hague University of Applied Sciences.

\section{Ulises Canchola Gutierrez}

has been the Ambassador of Mexico to the Kingdom of Norway since July 2019. A professional diplomat since 1993, he holds a bachelor's degree in law, and a master's degree in international law from the Fletcher School of Law and Diplomacy. He has specialised in international law and in multilateral diplomacy, serving in the Permanent Missions of Mexico to the United Nations in New York, Geneva and Vienna. As the Chairperson of the Personnel Commission of the Mexican Foreign Service, he directed the reform of the Mexican Foreign Service Law and its regulations in 2018-2019.

\section{José-Juan López-Portillo}

serves the Mexican Foreign Service as an external appointment as Counsellor in the Embassy of Mexico to the Kingdom of Norway. Before taking up this position, he was a Professor of International History at the Centro de Investigación y Docencia Económicas (CIDE) in Mexico City. He holds a PhD in Global History from the University of London, and is the author of several publications. 